\title{
Enunciación
}

\section{Estrategias tutoriales para la argumentación escrita en educación superior}

\author{
Tutorial Strategies for Written Argumentation \\ in Higher Education
}

Gloria Esperanza Mora-Monroy ${ }^{\circledR}{ }^{\circledR}$, Miguel Ángel Fuerte-Blanco ${ }^{\circledR}$

\section{Resumen}

El objetivo de este artículo es identificar y sistematizar las estrategias utilizadas por tutores para el desarrollo de la argumentación escrita de estudiantes de distintas carreras pertenecientes a los Cursos Nivelatorios de Lectoescritura en la Universidad Nacional de Colombia. La investigación se desarrolla desde un enfoque cualitativo de teoría fundamentada en los datos. La recolección de la información se ha realizado a través de registros de tutorías y sesiones de discusión llevadas a cabo con los tutores durante 2018, con el fin de reflexionar sobre su propia acción. Como resultados principales se identifican estrategias mayoritariamente de andamiaje cognitivo, y en menor medida, de carácter instructivo, clasificadas en estrategias para que el tutorado tome conciencia del carácter polémico de la argumentación, para la formulación de una tesis y para la búsqueda de argumentos pertinentes con la tesis.

Palabras clave: tutoría, estrategias tutoriales, argumentación escrita, enseñanza superior.

\begin{abstract}
This article aims to identify and systematize the strategies used by tutors for the development of written argumentation in students from different study programs in literacy leveling courses at Universidad Nacional de Colombia. This research is conducted from an approach based on Grounded Theory. Data collection was performed through recordings of tutoring and discussion sessions carried out with tutors during 2018 to reflect on their own performance. Strategies mostly involving cognitive scaffolding are identified as the main results, as well as instruction strategies to a lesser extent. Those strategies are classified into three groups depending on the tutor's purpose: awareness of the controversial nature of argumentation, formulation of a thesis, and search for appropriate arguments in relation to the thesis.
\end{abstract}

Keywords: tutoring, tutoring strategies, written argument, higher education.

1 Profesora asociada, departamento de Lingüística, Universidad Nacional de Colombia. Correo electrónico: gemoram@unal.edu.co. ORCID: https://orcid.org/0000-0001-6485-0504

2 Licenciado en español y filología clásica. Universidad Nacional de Colombia. Estudiante de la Licenciatura en Filología e Idiomas - Inglés. Correo electrónico: mafuerteb@unal.edu.co.

ORCID: https://orcid.org/0000-0003-3856-6088

Cómo citar: Mora-Monroy, G. E. y Fuerte-Blanco, M. A. (2021). Estrategias tutoriales para la argumentación escrita en educación superior. Enunciación, 26(2), 172-187. DOI: https://doi.org/10.14483/22486798.17776 


\section{Introducción}

Para superar las dificultades en relación con la escritura académica universitaria han surgido alternativas como la creación de políticas institucionales alrededor de la alfabetización académica, el ofrecimiento de cursos de escritura al comienzo de la vida universitaria, y la creación de programas o centros de escritura, cuyo acompañamiento es brindado por tutores pares o docentes (Calle Álvarez, 2016; Cardozo-Ortiz, 2011; Núñez Cortés, 2013, 2020; Redlees, 2015). En la Universidad Nacional de Colombia se ofrecen los Cursos Nivelatorios de Lectoescritura (CNLE) ${ }^{1}$, los cuales se han centrado en la lectura y escritura de textos argumentativos, por cuanto consideramos que la argumentación está estrechamente vinculada al desarrollo del pensamiento crítico de los estudiantes (Cassany y Castellà, 2010; Rapanta, 2019; Schwarz, 2009; Sanders et al., 2009), para desempeñarse en contextos universitarios, profesionales y cotidianos.

La escritura de este tipo de textos plantea varios retos para los estudiantes, como la formulación de preguntas y afirmaciones que impliquen un carácter polémico (Camps, 1995) o el planteamiento de argumentos pertinentes para defender una tesis. Igualmente, la enseñanza de la escritura argumentativa plantea desafíos para los docentes que pretendan apoyar el desarrollo de esta en sus estudiantes (Bañales Faz et al., 2015), tanto en cursos introductorios a la escritura universitaria como en los cursos disciplinares. Estos desafíos están relacionados con el qué, cómo y cuándo enseñar la argumentación escrita, de modo que los estudiantes puedan superar las dificultades que enfrentan y ganar autonomía en el abordaje de estas actividades de escritura. Para ayudar en la superación de estos retos, en los

1 En las sedes andinas (Bogotá, Medellín, Manizales, Palmira) este curso se imparte a aproximadamente el $10 \%$ de los estudiantes admitidos a primer semestre. En las sedes de presencia nacional (Amazonia, Caribe, Orinoquia, Tumaco) se ofrece a la totalidad de estudiantes que ingresan a primer semestre. En este artículo, se incluye información sobre lo que se ha hecho en el campo de la tutoría con estudiantes de la sede Bogotá. En esta sede cada uno de los grupos suele tener entre 25 y 30 estudiantes y se ofrecen entre seis y diez grupos cada semestre.
CNLE la actividad docente se ha apoyado en el trabajo de estudiantes de últimos semestres del área del lenguaje, quienes fungen como tutores pares de escritura para promover espacios de aprendizaje cooperativo (Azorín Abellán, 2018) y apoyar el proceso de escritura de un ensayo argumentativo de los estudiantes pertenecientes a estos cursos.

En relación con estrategias para la enseñanza de la escritura argumentativa desde la perspectiva del docente, se encuentran aportes en varios trabajos (Andrews, 2010; Andriessen y Baker, 2014; Bañales Faz et al., 2015; Díaz, 2002; Rapanta, 2019; Serrano de Moreno, 2008; Schwarz, 2009). Sin embargo, encontramos menos literatura en relación con estrategias para acompañar la escritura de textos argumentativos desde la perspectiva del tutor, a excepción de dos trabajos de pregrado (Castillo, 2019; Restrepo, 2018) y de una guía de preguntas para que el tutor evalúe textos argumentativos (Quintana, 2004, p. 29), así como avances en relación con estrategias tutoriales para favorecer procesos de escritura sin ahondar en un tipo de texto en particular (Brown, 2008; Chois-Lenis y Guerrero, 2015; Mackiewicz y Thompson, 2015; Núñez Cortés, 2020; Roldán Morales y Arenas Hernández, 2016).

En tal sentido, el objetivo de este artículo es identificar y sistematizar las estrategias que usan tutores asignados a los CNLE para ayudar a los estudiantes a superar las dificultades que la escritura argumentativa les demanda. Esta sistematización puede constituir un aporte en relación con el vacío reportado en la revisión de literatura.

\section{Referentes conceptuales y antecedentes}

\section{La enseñanza de la argumentación escrita en los CNLE}

Dentro de los referentes teórico-pedagógicos que guían la labor de docentes y tutores en los CNLE, tenemos en cuenta el enfoque de la enseñanza estratégica (Monereo, 2008), la cual tiene como objetivo que los estudiantes "sean capaces de 
autorregular sus acciones para aprender, [...] hacerlos más conscientes de las decisiones que toman, de los conocimientos que ponen en juego, de sus dificultades para aprender y del modo de superar estas dificultades" (p. 12). Esto se logra a través de procedimientos como la presentación y análisis de modelos que favorezcan procesos de metacognición, la construcción de preguntas, la práctica guiada y autónoma, así como el aprendizaje cooperativo (Azorín Abellán, 2018; Monereo, 2008). En los CNLE se analizan de manera conjunta y luego autónoma argumentaciones de la vida cotidiana y textos argumentativos en cuanto a su carácter polémico, el contexto de tal polémica, las posiciones de locutores e interlocutores y los argumentos planteados. El análisis de estos textos sirve como modelo para los propios procesos de escritura de los estudiantes, los cuales suelen girar en torno a temas de coyuntura nacional.

Como parte de la enseñanza estratégica de la escritura, en los CNLE también se tiene en cuenta el enfoque de escritura como proceso basado en la regulación que implica subprocesos de planeación, textualización y revisión (Castelló et al., 2010; Sala-Bubaré y Castelló, 2018). En cada uno de estos subprocesos el estudiante es guiado por los docentes y por los tutores. Estos últimos acompañan el proceso de manera más personalizada y se convierten en los primeros lectores de los textos de los estudiantes. Este espacio de lectura y revisión de pares, así como la posibilidad de publicar en la revista Prima Exagia², se dan en consonancia con el principio de que la lectura y la escritura son actividades discursivas contextualizadas (Camps, 2004; Carlino, 2002, 2006, 2013).

En nuestra práctica también cobra relevancia la perspectiva de la enseñanza y aprendizaje dialógicos en la que docentes y tutores aprenden formas de enseñar y los estudiantes enseñan formas de aprender (Aubert et al., 2009; Freire, 2002). En

2 La revista Prima Exagia es una publicación de carácter semestral coordinada por los tutores, quienes conforman el Grupo de Apoyo a los CNLE. Esta publicación recoge los mejores textos producidos en el marco de los CNLE (ver http://bienestar.bogota.unal.edu.co/pgp/Publicaciones/prima_exagia/ prima_exagia.html). conexión con este, adoptamos la noción de argumentación colaborativa (Nussbaum, 2021) en la que docentes, tutores y estudiantes trabajan juntos para construir y refutar argumentos más que para ganar las disputas (Nussbaum, 2021; Van Eemeren y Grootendorst, 2004). La argumentación colaborativa da relevancia a la construcción de andamiajes a través de preguntas para evaluar la validez de los argumentos y del uso de esquemas gráficos para sopesar argumentos y contraargumentos (Nussbaum, 2021).

En cuanto a la conceptualización de argumentación, tenemos en cuenta la argumentación como proceso de indagación (Bañales Faz et al., 2015; Sampson et al., 2011) y como instrumento para promover procesos de aprendizaje (Andrews, 2010; Andriessen y Baker, 2014; Molina y Carlino, 2013; Nussbaum, 2021; Rosales y Vázquez, 2011; Schwarz, 2009). También tomamos en consideración el modelo argumentativo de Toulmin (Toulmin et al., 1984) y los tipos de argumentos al igual que los errores y falacias argumentativas propuestas por Weston (2006).

Dado el interés de centrarnos en las estrategias que usan los tutores pares en su proceso de acompañamiento personalizado a la escritura del ensayo argumentativo, presentamos enseguida la conceptualización sobre la tutoría entre pares.

\section{La tutoría entre pares (peer tutoring)}

La tutoría entre pares es una modalidad de aprendizaje cooperativo personalizado, caracterizada por la relación asimétrica entre sus participantes: un estudiante con un nivel de competencia mayor en el área de interés apoya el proceso de otro menos experto (Alzate-Medina y Peña-Borrero, 2010; Chois-Lenis et al., 2017; Duran et al., 2014; Molina, 2017; Topping, 1996). Con esta, el tutor orienta el proceso formativo de un estudiante tutorado, con miras a promover su autonomía y el fortalecimiento de aspectos académicos, emocionales y profesionales (Alzate-Medina y Peña-Borrero, 2010), así como el favorecimiento de la integración del tutorado a la universidad (Gallego 
y Sánchez, 2016), a través del acompañamiento personalizado, el cual es mediado por el docente (Duran et al., 2014), quien le ayuda al tutor a adquirir las bases requeridas para su labor pedagógica (Cardozo-Ortiz, 2011). De esta manera, la tutoría entre pares no se restringe al ámbito académico, sino que propende por el desarrollo integral y la adaptación del estudiante (Arbizu et al., 2005). De acuerdo con Barberá (2006), desde una perspectiva socioconstructivista, el tutor es un participante importante en la construcción significativa de conocimientos por parte del tutorado, por cuanto favorece la generación de autonomía (Monereo, 2008) y el aprendizaje significativo a partir de la ayuda pedagógica que ofrece. En este proceso, tanto el tutor como el tutorado reportan la construcción de aprendizajes de carácter recíproco dada la relación establecida entre ambos (Durán y Flores, 2014; Villagra-Bravo y Valdebenito-Zambrano, 2019).

La tutoría entre pares como estrategia facilita la apropiación de las convenciones epistemológicas y discursivas de determinada disciplina por parte del tutorado, en tanto el estudiante tutor, debido a sus experiencias en escritura, puede aproximarse de manera más individual y cercana al estudiante, así como reconocer en este las confusiones que atraviesa (Moust y Schmidt, citados por Chois-Lenis et al., 2017; O'Neill, 2008). Por este motivo, el objetivo de la tutoría es el desarrollo de estrategias de fortalecimiento de los procesos metacognitivos asociados a la escritura a través del diálogo y la construcción colectiva, los cuales forman parte de la enseñanza estratégica (Monereo, 2008).

\section{Estrategias tutoriales}

Con miras a facilitar el aprendizaje del tutorado acerca de la argumentación escrita, los tutores emplean diferentes estrategias didácticas, entendidas como "procedimientos que el agente de enseñanza utiliza en forma reflexiva y flexible para promover el logro de aprendizajes significativos en los estudiantes" (Tebar, 2003, p. 7). Para esta investigación, establecemos diálogo con el marco elaborado por Mackiewicz y Thompson (2015), cuyas bases teóricas se encuentran en la zona de desarrollo próximo de Vygotski (2009) y el andamiaje (scaffolding) (Wood et al., 1976). Estas autoras dividen las estrategias en tres grupos: instrucción (instruction), andamiaje cognitivo (cognitive scaffolding) y andamiaje motivacional (motivational scaffolding).

Las estrategias de instrucción son aquellas en las cuales el tutor propone opciones o plantea soluciones de manera directiva, en lugar de crear las condiciones para que el estudiante Ilegue a la solución o a la respuesta por sí mismo. En este grupo, Mackiewicz y Thompson (2015) proponen tres estrategias: decir, sugerir y explicar, y dar ejemplos. En las dos primeras, el tutor se dirige al estudiante para informar, realizar sugerencias o brindar consejos. La diferencia radica en la directividad del discurso: mientras que en la primera hay nula o poca mitigación de esta directividad, en la segunda sí la hay. La tercera, por su parte, hace referencia a la elaboración e ilustración por parte del tutor acerca de un tema determinado.

En segundo lugar, las estrategias de andamiaje cognitivo son aquellas en las que el tutor ayuda al estudiante a determinar una respuesta, corregir un error o ejecutar una tarea a través del diálogo y la pregunta, sin hacerlo por él. En este grupo se incluyen ocho estrategias tutoriales: formular preguntas, leer en voz alta, responder como lector u oyente, referirse a un tema presentado anteriormente, forzar una elección, incitar, insinuar y demostrar.

En tercer lugar, Mackiewicz y Thompson (2015) proponen las estrategias de andamiaje motivacional, que buscan crear una relación de afinidad, solidaridad y confianza en la que el tutorado se sienta seguro y participe de manera activa en su proceso de aprendizaje. Las cinco estrategias que componen ese grupo son: mostrar interés, elogiar (retroalimentación positiva y recompensas verbales), reforzar la apropiación y control del estudiante sobre su proceso, ser optimista o utilizar humor, y mostrar simpatía o empatía. 
Tabla 1. Estrategias tutoriales de Mackiewicz y Thompson (2015)

\begin{tabular}{|c|c|}
\hline Tipo de estrategia & Estrategia \\
\hline \multirow{3}{*}{ Instrucción } & Decir \\
\hline & Sugerir \\
\hline & Explicar y dar ejemplos \\
\hline \multirow{8}{*}{ Andamiaje cognitivo } & Formular preguntas \\
\hline & Leer en voz alta \\
\hline & Responder como lector u oyente \\
\hline & Referirse a un tema presentado anteriormente \\
\hline & Forzar una elección \\
\hline & Incitar \\
\hline & Insinuar \\
\hline & Demostrar \\
\hline \multirow{5}{*}{ Andamiaje motivacional } & Mostrar interés \\
\hline & Elogiar \\
\hline & Reforzar la apropiación y control del estudiante sobre su proceso \\
\hline & Ser optimista o utilizar humor \\
\hline & Mostrar simpatía o empatía \\
\hline
\end{tabular}

Fuente: elaboración propia.

\section{Metodología}

Esta investigación se llevó a cabo desde la teoría fundamentada en los datos, en la que el interés se centra en tratar de "comprender el significado o naturaleza de la experiencia de las personas" (Strauss y Corbin, 2002, p. 20). En nuestro caso, nos interesan las significaciones que los tutores de los CNLE dan a su propia actividad de tutoría, con el ánimo no solo de comprenderla, sino también de poder evaluarla para su eventual transformación (Camps, 2004). Además, recurrimos a la teoría fundamentada en los datos ante la escasa literatura relacionada con estrategias para favorecer procesos de argumentación escrita desde la perspectiva del tutor en particular. Buscamos hacer un ordenamiento conceptual por el cual organizamos y clasificamos, como lo proponen Strauss y Corbin (2002), las estrategias usadas por los tutores como parte de la construcción de andamiajes para apoyar los procesos de escritura de textos argumentativos de los estudiantes. Los resultados de la identificación de estas estrategias pueden convertirse en un apoyo para la acción social (Strauss y Corbin, 2002) de otros tutores que apoyan este mismo tipo de procesos.

\section{Participantes y tipos de datos}

Los participantes en esta investigación son estudiantes de últimos semestres de programas del área del lenguaje, quienes fungen como tutores de los estudiantes que toman los cursos. Estos tutores disponen de espacios externos al horario de clase para realizar acompañamiento personalizado o en pequeños grupos a los estudiantes. Además, asisten a las clases y apoyan a los tutorados dependiendo de las dinámicas y actividades de los docentes.

El lapso sobre el que se realizan los análisis corresponde a dos periodos académicos de 2018, a saber: 2018-01, en el que participaron cuatro tutores que apoyaron ocho cursos, y 2018-02, con cuatro tutores para siete cursos $^{3}$. Para identificar las estrategias utilizadas por los tutores en el acompañamiento a los procesos de escritura de textos argumentativos, hemos tenido en cuenta: a) registros de tutorías a través de formularios de Google ${ }^{4}$ : trece

3 Cuando un tutor apoya un solo curso cumple también con las labores de coordinar a los demás tutores y liderar la publicación de la revista Prima Exagia.

4 En estos formularios, los tutores indican fecha, nombres de los tutorados, semestre que cursan, carrera, grupo al que pertenecen; si el estudiante acude por su cuenta o por sugerencia del (de la) docente, propósito de la tutoría, contenidos desarrollados (procedimentales, conceptuales, 
documentadas en el 2018-01 y 43 en el $2018-02^{5} ;$ b) doce sesiones de discusión llevadas a cabo entre los tutores, moderadas por la coordinadora de los CNLE durante los dos periodos académicos. Estas sesiones, de carácter semiestructurado, se realizaron con el objetivo de conversar y reflexionar sobre la propia práctica tutorial, teniendo en cuenta que, como se afirma desde la teoría fundamentada (Strauss y Corbin, 2002), las intenciones y acciones de los tutores se pueden definir y redefinir por la interacción entre unos y otros. Estas sesiones de discusión tuvieron un carácter natural, por cuanto no surgen por iniciativa del investigador (Galeano, 2004) para realizar esta investigación particular, sino que se han venido realizando desde antes de 2018. Tanto los registros de tutorías analizados como las sesiones de discusión corresponden a una muestra por conveniencia, basada en la proximidad de los participantes con los investigadores (Mendieta Izquierdo, 2015).

\section{Procedimiento de análisis}

El análisis de los datos se llevó a cabo con el programa NVIVO y con procedimientos como la codificación de información a través de palabras clave para identificar estrategias comunes o patrones, guiados siempre por la pregunta acerca de cómo se relacionan estos datos con los anteriores (Freeman, 1998) y qué tan recurrentes son de modo que se puedan convertir en categorías. Así surgió la clasificación de estrategias a partir de las fases más importantes en la planeación de los textos argumentativos. Este análisis implicó procesos de codificación abierta, en el que identificamos categorías recurrentes y de codificación axial (ver "Tendencias en las estrategias encontradas"), en el que establecimos relaciones entre las categorías (Strauss y Corbin, 2002). Partir de los datos no significa que no establecimos interacciones con la teoría construida anteriormente, pues las clasificaciones hechas por Mackiewicz y Thompson (2015)

actitudinales), desarrollo metodológico, acuerdos con el estudiante y si hay uso de materiales didácticos estos se deben adjuntar.

5 En el primer semestre, los tutores hicieron pocos registros de tutorías, posiblemente porque esta fue una labor que se comenzó a realizar por primera vez en ese semestre. también nos permitieron llegar a generalizaciones importantes en relación con la clasificación de las estrategias.

\section{Resultados y discusión}

En relación con la tarea comunicativa a la que apuntan estos cursos a lo largo del semestre, la elaboración de un texto argumentativo, los tutores reportan múltiples dificultades por parte de los estudiantes ${ }^{6}$. Centramos nuestra presentación en tres categorías que han surgido del análisis de los datos, especialmente para las etapas de planeación y organización de la información del texto argumentativo: a) las estrategias para ayudar a superar las dificultades relacionadas con entender el carácter polémico de la argumentación, b) para ayudar en la construcción de una tesis y c) para la búsqueda de argumentos en favor de una tesis.

Como ya señalamos, para la agrupación de las estrategias tutoriales también fue útil la clasificación hecha por Mackiewicz y Thompson (2015) en estrategias de carácter directivo/instructivo, de andamiaje cognitivo y de andamiaje motivacional. No obstante, en nuestro análisis encontramos nuevas estrategias que las autoras no incluyen dentro de estas categorías, por cuanto nos concentramos en estrategias para favorecer procesos de argumentación.

\section{Estrategias tutoriales para ayudar a tomar conciencia sobre el carácter polémico de la argumentación}

Una de las principales dificultades que los tutores reportan en relación con la construcción de textos argumentativos es formular preguntas y afirmaciones que efectivamente impliquen un carácter polémico o de debate. Este tipo de dificultades también

\footnotetext{
6 Estas van desde los niveles más microestructurales, como la ortografía, la construcción de oraciones, el uso de signos de puntuación, la selección léxica, el manejo de fuentes y de citas textuales, hasta problemas relacionados con los procesos de planeación de la argumentación como tal. Aquí nos centramos en las estrategias para este tipo de dificultades más globales relacionadas con la formulación y organización de las ideas en el texto argumentativo.
} 
ha sido planteado por autores como Camps (1995) y Bañales Faz et al. (2015). Mostramos a continuación cinco estrategias identificadas en orden de mayor a menor frecuencia de aparición, de acuerdo con el análisis hecho en NVIVO. En la tabla 2 se resumen las frecuencias de aparición.

1. La primera consiste en discutir sobre la pregunta que el estudiante toma como punto de partida para la planeación y escritura de su texto, y sobre el tipo de texto que se proyecta a partir de ella, esto es, argumentativo o expositivo: "entonces, claro, a veces pregunta... a veces dentro de sus preguntas había un '¿Cómo es que tal y cual y cual?'. Y yo: 'Me dices un 'cómo', ya no, ya no estás haciendo un texto argumentativo, ya va a ser explicativo, informativo, pero no va a ser argumentativo'" (Elementos internos $\backslash \backslash$ Reuniones $\backslash \backslash$ Monitores 1-23/02/18).

2. La segunda estrategia es proponer debates en los que el estudiante se enfrente a un público real para tratar de convencerlo de una postura, estrategia común utilizada por las docentes y también por los tutores a través de la implementación de talleres en clase:

[...] eso es lo que pasa en el taller de falacias, precisamente, que se le pone al estudiante a defender ante todos una posición polémica, generalmente llegando ya al absurdo, ¿no? Y ahí se dan cuenta de que efectivamente la tesis tiene que ser algo controversial y que se encuentran con un público que va a buscar todas las herramientas posibles para desvirtuar sus argumentos. (Elementos internos $\backslash \backslash$ Reuniones $\backslash \backslash$ Monitores 1-23/02/18)

3. La tercera estrategia para familiarizar al estudiante con el carácter polémico de la argumentación es identificar si el planteamiento formulado inicialmente como tesis puede contraponerse con otras posturas o no, para lo cual los tutores proponen imaginarse un público posible al que se debe convencer de una postura o determinar si de lo propuesto como tesis es posible decir: "estoy o no estoy de acuerdo con ello". Así lo vemos en el siguiente caso en el que interactúan dos tutores:

Tutor 1: Entonces yo les decía: "bueno, dame una aseveración de cualquier cosa, que no sea de esto" y me decía: "el vino puede emborrachar", ¿no? Así, como de pronto, y yo: "entonces, dímelo, dime, dime una afirmación cualquiera, es para <ininteligible $>$. Di una afirmación cualquiera, la que sea". Tutor 2: Ehhh, no sé, el sistema de transporte bogotano es un asco.

Tutor 1: No estoy de acuerdo.

Tutor 2: Sí, ese es el punto.

Tutor 1: No estoy de acuerdo, y entonces, y entonces claro...

$<$ Cruce de voces $>$

Tutor 1: No estoy de acuerdo y dices algo que no estás de acuerdo, te lo voy a demostrar y te voy a poner todos los argumentos, eso, y yo les ejemplificaba así, entonces ellos ya "ahhh, claro". Eso es lo que tienes que... (Elementos internos $\backslash \backslash$ Reuniones $\backslash \backslash$ Monitores 1-23/02/18)

Consideramos que la mayoría de estas estrategias pueden ser de andamiaje cognitivo o de tipo directivo en tanto menor o mayor sea la intervención por parte del tutor; esto es, en tanto el tutor promueva más el trabajo activo por parte del estudiante, menor nivel directivo tendrá la tutoría y más carácter de andamiaje cognitivo. Sin embargo, la estrategia de involucrar a los estudiantes en debates es claramente de andamiaje cognitivo.

En la tabla 2 se resumen las estrategias para favorecer la concientización por parte del estudiante sobre el carácter polémico de la argumentación.

\section{Estrategias tutoriales para la construcción de tesis}

La tesis constituye la columna vertebral de cualquier texto argumentativo, por lo cual es importante construir estrategias que permitan a los estudiantes entender mejor en qué consiste su formulación. A partir del análisis se han identificado 
Tabla 2. Estrategias tutoriales para concientización sobre el carácter polémico de la argumentación

\begin{tabular}{cllc}
\hline \multicolumn{1}{c}{$\begin{array}{c}\text { Propósito de la } \\
\text { estrategia }\end{array}$} & \multicolumn{1}{c}{ Estrategia } & \multicolumn{1}{c}{$\begin{array}{l}\text { Clasificación según categorías de } \\
\text { Mackiewicz y Thompson (2015) }\end{array}$} & Frecuencia \\
\hline \multirow{3}{*}{ Carácter polémico } & $\begin{array}{l}\text { Discusión pregunta y texto que se } \\
\text { proyecta } \\
\text { Debates } \\
\text { Encontrar posturas contrarias }\end{array}$ & Andamiaje cognitivo/Instruccional & 5 \\
& Andamiaje cognitivo & 3 \\
& Andamiaje cognitivo/Instruccional & 3 \\
\hline
\end{tabular}

Fuente: elaboración propia.

cinco estrategias al respecto, las cuales también se presentan de acuerdo con su frecuencia en los registros de tutorías e intervenciones de los tutores (ver tabla 3).

1. Uno de los aspectos más relevantes para que los estudiantes entiendan en qué consiste la formulación de una tesis es que esta no surge por sí sola, sino en relación con ciertos problemas o situaciones problemáticas que le dan contexto (Baquero y Pardo, 1997). Los tutores también reportan que los estudiantes tienen dificultades para distinguir entre una tesis y un tema. Por tanto, una estrategia que varios de ellos reportan consiste en contrastar el tema, el problema, la tesis y la pregunta, lo cual puede ser de tipo directivo cuando el tutor mismo proporciona ejemplos al respecto, o de andamiaje cognitivo cuando las respuestas se van construyendo entre juntos. En el siguiente ejemplo, encontramos una interacción entre lo directivo y el andamiaje cognitivo:

De manera que iniciamos con la importancia de tener clara la problemática, de ahí surgirían dos o más posturas de las cuales el estudiante debía defender una. Él tenía clara su postura, pero no tenía plena claridad de en qué punto se ligaba a una problemática o cómo defenderla de ahí en adelante. (Archivos $\backslash \backslash$ Registro tutorías $\backslash \backslash$ Registro tutorías 2018-2).

2. Una segunda estrategia es verificar la intención del estudiante invitándolo a revisar la redacción de la tesis o a que parafrasee su tesis en los términos más coloquiales posibles, estrategia de andamiaje cognitivo:

Para solucionar este problema, intenté que me explicase con sus propias palabras aquello que realmente quería defender en su ensayo, de la forma más sencilla y coloquial posible. Una vez lo hizo, y yo quedé seguro de qué era realmente lo que deseaba para su ensayo, le orienté para que fuese capaz de crear una tesis de acuerdo a sus intenciones discursivas. (Elementos internos $\backslash \backslash$ Registro tutorías $\backslash \backslash$ Registro tutorías 2018-2)

Este tipo de estrategias están relacionadas con actividades que desde los inicios de la perspectiva de escribir para aprender fueron propuestas para alentar a los estudiantes a pensar sobre el papel a través de los usos más expresivos de la escritura, entre ellos poder conversar en relación con las temáticas sobre las que después se solicita escribir (Vacca y Linek, 1992).

3. Una tercera estrategia es ayudar al estudiante a establecer conexiones entre la temática propuesta por su docente y sus intereses personales, estrategia que ubicamos como andamiaje motivacional:

Un aspecto que considero muy importante en la construcción de la tesis es el interés del estudiante. Si bien en los cursos que acompañé había un tema al que se debían ceñir los estudiantes (memoria), en esta tutoría y en otras, procuré ayudarlos a encontrar primero su tema de interés con la convicción personal de que cualquier tema se podía articular con el 
tema de memoria. Este estudiante empezó diciendo que no sabía nada del tema de memoria y que por eso se le dificultaba, pero luego encontró que este se podía relacionar con la comunidad LGBT, tema que le apasionaba y del cual sabía bastante. (Elementos internos $\backslash \backslash$ Registro tutorías $\backslash \backslash$ Registro tutorías 2018-1)

Si bien Mackiewicz y Thompson (2015) definen las estrategias de andamiaje motivacional como aquellas que se utilizan para generar confianza entre tutor y tutorado, también creemos que estas se pueden orientar hacia la motivación del estudiante con respecto a los temas sobre los cuales se le propone trabajar. Este tipo de estrategias resulta coincidente con lo señalado por Quintana (2004), en el sentido de que también es responsabilidad del tutor hacer que el estudiante disfrute del proceso de redacción, aprecie su importancia y adquiera estrategias.

4. La siguiente estrategia tiene que ver con que algunos estudiantes llegan a la tutoría sin la claridad suficiente sobre el concepto mismo de tesis y algunos criterios para su construcción. Por ello, es necesario reforzar este concepto a través de la explicación, estrategia de tipo instruccional: "Cuando lo logré, y no me quedaron dudas de que lo había entendido, le ofrecí indicaciones sobre cómo crear una buena tesis y cómo esta podía ser apoyada por argumentos" (Elementos internos $\backslash \backslash$ Registro tutorías $\backslash \backslash$ Registro tutorías 2018-2).

5. La última estrategia identificada para apoyar la construcción de la tesis es formular preguntas, por ejemplo, para indagar qué sabe el estudiante del tema sobre el que pretende escribir, y eventualmente invitarlo a profundizar sobre la temática:

El problema es que cuando llegan los estudiantes, digamos por ejemplo, en ese, en ese tema puntual de la tesis, Ilegan a tutoría, la tutoría sí es un poco, cómo explicarlo, como un poco ahondar en qué sabe el estudiante del tema, porque como lo decían aquí en uno de los manuales, es muy difícil que alguien empiece a escribir de algo que no sabe, entonces mi primer, mi primer acercamiento a los estudiantes es realmente "sabes del tema, has leído, entiendes que para que puedas formular una tesis debes tener unas lecturas..." (Elementos internos $\backslash \backslash$ Reuniones $\backslash \backslash$ Monitores 8-01/10/18)

Este tipo de estrategias coincide con uno de los principios que guía la práctica de los CNLE: escribir textos argumentativos implica indagar sobre las distintas posturas en relación con el tema que al estudiante le interesa para poner a prueba sus propios puntos de vista (Weston, 2006) y tomar posturas mejor informadas al respecto. En otros términos, argumentar también es una oportunidad de usar la escritura para aprender (Andrews, 2010; Andriessen y Baker, 2014; Molina y Carlino, 2013; Nussbaum, 2021; Rapanta, 2019; Rosales y Vázquez, 2011). Al respecto, Schwarz (2009) explica cómo al tiempo que se puede aprender a argumentar, el argumentar mismo es una oportunidad para aprender.

Una tendencia que se evidencia en las estrategias orientadas a guiar el proceso de construcción de la tesis es la búsqueda de claridad tanto en la conceptualización acerca de las características de una tesis como en las ideas que desarrolla el tutorado en relación con su temática de interés. Para el primer efecto los tutores pueden recurrir a estrategias instructivas tales como reforzar el concepto, mientras que para el segundo usan estrategias de andamiaje cognitivo, de modo que el estudiante vaya ganando claridad sobre el tema y la postura que desea sustentar en su texto.

En la tabla 3 se resumen las estrategias identificadas para apoyar a los estudiantes en la construcción de sus tesis.

\section{Estrategias tutoriales para la búsqueda de argumentos}

Una vez los estudiantes logran tener más clara una tesis para sustentar, la siguiente tarea en el proceso es que puedan encontrar argumentos realmente pertinentes para dar soporte a sus posturas. Las 
Tabla 3. Estrategias tutoriales para la construcción de la tesis

\begin{tabular}{lllc}
\hline $\begin{array}{c}\text { Propósito de la } \\
\text { estrategia }\end{array}$ & \multicolumn{1}{c}{ Estrategia } & \multicolumn{1}{c}{$\begin{array}{c}\text { Clasificación según categorías de } \\
\text { Mackiewicz y Thompson (2015) }\end{array}$} & Frecuencia \\
\hline & Contrastar tema-tesis-pregunta problema & Andamiaje cognitivo/Instruccional & 14 \\
& $\begin{array}{l}\text { Parafrasear/revisar redacción tesis con } \\
\text { Construcción de la }\end{array}$ & Andamiaje cognitivo & 9 \\
tesis & $\begin{array}{l}\text { Conectar temática con interés particular } \\
\text { Reforzar concepto }\end{array}$ & Andamiaje motivacional & 4 \\
& Formular preguntas & Andamiaje instruccional & 4 \\
& Indagación temática & Andamiaje cognitivo & 2 \\
\hline
\end{tabular}

Fuente: elaboración propia.

siguientes son las estrategias reportadas para apoyar esta parte del proceso.

1. La estrategia mayormente señalada es invitar a reelaborar los argumentos de acuerdo con criterios como la claridad de cada uno, las relaciones entre uno y otro, y especialmente la relación entre estos y la tesis. Una de las estrategias al respecto es identificar los garantes (Toulmin et al., 1984), marcos conceptuales o creencias (Pardo y Baquero, 2001) que están a la base de la relación entre tesis y argumentos:

Se le solicitó al estudiante identificar sus argumentos, señalando la conclusión de cada uno de sus argumentos, sus datos y garantes. A partir de esto, se pudo determinar que dos de sus argumentos terminaban con conclusiones que no eran el resultado de los datos y garantías con los que se contaba. Por ende, esto afectaba la coherencia de sus ideas. (Elementos internos $\backslash \backslash$ Registro tutorías $\backslash \backslash$ Registro tutorías 2018-2)

2. La segunda estrategia mayormente reportada es volver a la tesis y eventualmente replantearla, de modo que haya coherencia entre esta y los argumentos planteados:

También me percaté de que sus argumentos no apoyaban plenamente la tesis. No obstante, tras debatirlo con él, nos dimos cuenta de que en realidad el problema no eran los argumentos, sino que no había sabido utilizar bien las palabras en la tesis para expresar lo que realmente quería. (Elementos internos $\backslash \backslash$ Registro tutorías $\backslash \backslash$ Registro tutorías 2018-2)

3. La tercera estrategia mencionada por los tutores es representar la estructura argumentativa a través de organizadores gráficos, como por ejemplo la red argumentativa (Pardo y Baquero, 2001), Ios cuales pueden facilitar una rápida visualización de los contenidos, su interiorización y recordación:

Se acuerda revisar la información que ya se tiene para poder seleccionar la más relevante y replantear los argumentos señalando cada una de las partes, según el modelo de Toulmin. Aunque esta última parte se encuentra en el formato, los estudiantes la omiten o la completan de una forma inadecuada. Sin embargo, cuando se les recuerda el esquema gráfico de la red y se les pide ubicar cada elemento, se ven forzados a revisar sus apuntes o a hacer preguntas frente a lo que no tienen claro, de modo que es más fácil irles explicando el modelo y, a su vez, encontrar las debilidades argumentativas. En consecuencia, se le pide revisar de la misma manera los demás argumentos y graficarlos en la red argumentativa que plantea el modelo. (Elementos internos $\backslash \backslash$ Registro tutorías $\backslash \backslash$ Registro tutorías 2018-2)

Cabe aclarar que en este fragmento se presenta una interacción de estrategias, pues si bien el tutorado es quien grafica la estructura 
detallada de uno de sus argumentos (andamiaje cognitivo), parece haber una explicación previa de la gráfica (estrategia directiva), seguida de una sugerencia para revisar los otros argumentos con la misma estrategia.

4. Otra estrategia es formular preguntas clave, bien para que el estudiante cualifique los argumentos y su conexión con la tesis, o bien para que identifique la necesidad de continuar indagando sobre el tema para fortalecer la argumentación presentada:

Al identificar que los argumentos que trae la estudiante no están realmente argumentando su tesis, puesto que no tienen garantías, se le pide a la estudiante leer su argumento y responder lo siguiente: ¿En qué se soporta tal aseveración?, ¿en qué información se basa?, ¿esos soportes realmente sustentan el tema o podrían llegar a ser irrelevantes para el contexto?, ¿por qué?, ¿cómo, partiendo de esa idea, llegamos a tal conclusión?, y ¿cuál es la información general que está detrás que nos permite llegar a tal punto? (Elementos internos $\backslash \backslash$ Registro tutorías $\backslash \backslash$ Registro tutorías 2018-2)

Como se evidencia en las estrategias encontradas para la búsqueda de argumentos, el tutor propende por que el tutorado genere y reconozca las relaciones entre los componentes internos del argumento (aserción, evidencias y garantes) y entre cada uno de los argumentos y la tesis que pretende defender. La tendencia por parte del tutor es utilizar estrategias de andamiaje cognitivo para que el estudiante tome un rol activo y se apropie de su proceso de aprendizaje. Esto se corresponde con la función del tutor en un contexto de tutoría entre pares, ya que funge como una audiencia preliminar, crítica y atenta, que favorece el desarrollo metacognitivo y metalingüístico del estudiante, así como el uso de estrategias de mejoramiento que distan de la mera corrección autoritaria del texto (Carlino, 2002).

A continuación, presentamos la tabla que resume las estrategias tutoriales encontradas para acompañar a los estudiantes en la definición de los argumentos para sus tesis.

\section{Tendencias en las estrategias encontradas}

Hasta aquí hemos presentado lo correspondiente a la codificación de primer nivel de los datos encontrados. En cuanto a la codificación axial, la comparación entre las estrategias presentadas en las secciones anteriores muestra una mayor tendencia de parte de los tutores a utilizar estrategias de andamiaje cognitivo. El predominio de estas estrategias resulta consistente con un escenario de tutoría en el que el estudiante debe ser el centro del proceso de aprendizaje y el acompañamiento debe estar orientado a brindarle herramientas que le permitan reconocerse como aprendiz, ser autónomo en su proceso y participar de manera activa

Tabla 4. Estrategias didácticas para la búsqueda de argumentos

\begin{tabular}{|c|c|c|c|}
\hline $\begin{array}{c}\text { Propósito de la } \\
\text { estrategia }\end{array}$ & Estrategia & $\begin{array}{l}\text { Clasificación según categorías de } \\
\text { Mackiewicz y Thompson (2015) }\end{array}$ & Frecuencia \\
\hline \multirow{4}{*}{ Búsqueda de argumentos } & $\begin{array}{l}\text { Reescribir argumentos e identificar } \\
\text { garantes }\end{array}$ & Andamiaje cognitivo & 8 \\
\hline & Replantear tesis & Andamiaje cognitivo & 6 \\
\hline & Graficar estructura argumentativa & Andamiaje cognitivo & 4 \\
\hline & $\begin{array}{l}\text { Formular preguntas. Invitar indaga- } \\
\text { ción temática }\end{array}$ & Andamiaje cognitivo & 4 \\
\hline
\end{tabular}

Fuente: elaboración propia. 
en este (Duran et al., 2014). En dicho escenario de tutoría se busca que el estudiante vea la escritura como un proceso y que fortalezca su confianza frente a esta práctica, y no que se convierta en un espacio en el que el tutor corrige la producción escrita del tutorado (Bell, citado por Topping, 1996; Carlino, 2006; Harris, 1995; Molina, 2017; Quintana, 2004).

La comparación entre las estrategias para la formulación de la tesis y para la búsqueda de argumentos muestra que en ocasiones los estudiantes requieren refuerzos en su comprensión sobre las características propias de la argumentación. Para este efecto, los tutores pueden recurrir a estrategias directivas, a través de la explicación de cómo se construye una tesis, $u$ ofrecer ejemplos que ilustren las diferencias entre argumentos y garantes. En tal sentido, Mackiewicz y Thompson (2015), respecto a la estrategia instructiva de explicar, sugieren que esta les permite a los estudiantes entender el porqué de determinada sugerencia u observación, más que solo el cómo seguirla, con lo que finalmente las estrategias directivas también contribuyen para favorecer la autonomía del tutorado.

Por otra parte, en el proceso de planear y construir sus textos argumentativos, los estudiantes necesitan ir ganando claridad sobre los contenidos mismos de su texto, es decir, sobre la temática particular que van a trabajar, la tesis que van a sustentar y los argumentos que van a utilizar. Para este efecto, los tutores usan estrategias de andamiaje cognitivo. En estos contextos específicos, la participación del tutorado es activa, en tanto la tutoría es un escenario de diálogo y confrontación de ideas en el que las subjetividades de ambos participantes "establecen negociaciones para definir el objetivo que se construirá de manera colaborativa" (Molina, 2017, p.18). Así, en esta situación comunicativa, es importante tanto la comprensión del estudiante como la del tutor para guiar de manera adecuada el proceso de planeación en concordancia con el propósito que persigue el estudiante con su texto.
Las estrategias encontradas, que parten de dificultades percibidas por el tutor tanto en la etapa de planeación como de escritura del primer borrador, sitúan a este como una figura mediadora que orienta el desarrollo de capacidades metacognitivas (Carlino, 2002) en el estudiante: evaluar los resultados del proceso, comparar el resultado con la planeación realizada y analizar los componentes del esquema argumentativo con miras a establecer relaciones coherentes.

Adicionalmente, la comparación entre las estrategias para la construcción de la tesis y la búsqueda de argumentos muestra que en ambas fases del proceso los tutores invitan a los estudiantes a reescribir y a graficar relaciones. En tal sentido, se promueve el uso de la escritura para que los estudiantes mejoren su comprensión en relación con la temática y subtemáticas sobre las que desean escribir, con lo cual la escritura adquiere el valor epistémico que debe tener (Carlino, 2013). Además, la reescritura en ambas etapas del proceso promueve que el tutorado sea claro consigo mismo y con el tutor, con quien forma una relación basada en el diálogo, para dar cuenta de sus ideas, lo cual resulta coincidente con los resultados de Chois-Lenis et al. (2017) sobre los beneficios que reporta la tutoría para los estudiantes.

Para finalizar, queremos señalar que la sistematización de las estrategias que los tutores de los CNLE usan en el acompañamiento a los estudiantes contribuye a la caracterización del papel que aquellos cumplen para favorecer el desarrollo de las competencias de argumentación escrita de los estudiantes. Sin embargo, esta investigación también nos ha permitido reflexionar sobre la práctica pedagógica de los mismos docentes por dos razones.

En primer lugar, porque nos permite identificar la distancia entre nuestros objetivos de enseñanza -la zona de desarrollo potencial de Vygotski (2009) - y el nivel en el que se encuentran los estudiantes -su zona de desarrollo efectivo-, debido a la mayor cercanía que los tutores tienen con los procesos particulares de los estudiantes y los 
diversos tipos de dificultades que se les pueden presentar en los niveles cognitivo y afectivo-emocional. Tal cercanía y relación intermedia ha sido reportada ya por varios autores. Así, por ejemplo, Topping (1996) señala que la relación entre tutor y tutorado se diferencia notablemente de la relación entre profesor y estudiante, en la medida en que la tutoría no consiste en la transmisión de conocimiento de una persona más hábil a otra menos hábil. Asimismo, Harris (1995) menciona que el tutor es una figura intermedia entre el profesor y el estudiante, lo que le permite acercarse a este último y trabajar de maneras que el profesor no puede.

En segundo lugar, el trabajo de los tutores también puede ayudar a enriquecer y mejorar la práctica de los docentes gracias a la identificación de la rica variedad de estrategias utilizadas por ellos en sus procesos de acompañamiento con los estudiantes. Adicionalmente, estas estrategias han surgido de la identificación de las principales dificultades por las que atraviesan los estudiantes, las cuales no siempre podemos distinguir los docentes al tener que orientar nuestra acción hacia los grupos completos.

\section{Conclusiones}

Los hallazgos de esta investigación revelan que en la práctica de los tutores de los CNLE para favorecer procesos de argumentación escrita predominan estrategias de andamiaje cognitivo, situación consistente con la creación de espacios en que se favorezca la autonomía por parte de los tutorados (Alzate-Medina y Peña-Borrero, 2010; Gallego y Sánchez, 2016) y con procesos pedagógicos de carácter (socio)constructivista. No obstante, las dificultades reportadas en relación con nociones como tesis o distinción entre tipos de argumentos, o entre argumentos y garantes, hacen necesaria la implementación de estrategias de tipo instruccional como el reforzamiento de ciertos conceptos o la presentación de ejemplos o analogías por parte de los tutores, útiles para los estudiantes con más dificultades.
Así mismo, los resultados de esta investigación constituyen un aporte en el campo de las estrategias tutoriales para favorecer procesos de escritura en la universidad, específicamente de textos argumentativos. La sistematización de las estrategias de andamiaje cognitivo y de carácter directivo en las distintas etapas de la argumentación, a saber: la identificación de temas polémicos, la construcción de tesis y la búsqueda de argumentos, se pueden convertir en un apoyo tanto para la formación de tutores de cursos introductorios a la lectura y escritura en la universidad, como de tutores ubicados en centros de escritura. Queda pendiente la identificación de estrategias de andamiaje motivacional, que también reportan los tutores, pero que por razones de espacio no pudieron ser desarrolladas en este artículo.

\section{Reconocimientos}

En este artículo se presentan resultados parciales de la investigación "Naturaleza de las propuestas didácticas en los Cursos Nivelatorios de Lectoescritura (CNLE), sede Bogotá, y su impacto en el desarrollo de la argumentación escrita", financiada por la Vicedecanatura de Investigación y Extensión de la Facultad de Ciencias Humanas, Universidad Nacional de Colombia, Convocatoria Orlando Fals Borda 2017, código 39018. Los autores agradecen a Johanna Acosta, Natalia Alejandra Leiton, Cristian Leonardo Murillo y Juan Alonso Mejías, quienes fungieron como tutores de los CNLE durante 2018. A partir de su trabajo y reflexiones se construyó este artículo.

\section{Referencias bibliográficas}

Alzate-Medina, G. M. y Peña-Borrero, L. B. (2010). La tutoría entre iguales: una modalidad para el desarrollo de la escritura en la educación superior. Universitas Psychologica, 9(1), 123-138.

https://doi.org/10.11144/Javeriana.upsy9-1.timd 
Andrews, R. (2010). Argumentation in higher education: Improving practice through theory and research. Routledge.

Andriessen, J. y Baker, M. (2014). Arguing to learn. En R. K. Sawyer (ed.), The Cambridge handbook of the learning sciences (pp. 439-460). Cambridge University Press. https://doi.org/10.1017/ CBO9781139519526.027

Arbizu, F., Lobato, C. y Del Castillo, L. (2005). Algunos modelos de abordaje de la tutoría universitaria. Revista de Psicodidáctica, 10(1), 7-21. https://ojs.ehu. eus/index.php/psicodidactica/article/view/367

Aubert, A., García, C. y Racionero, S. (2009). El aprendizaje dialógico. Cultura y Educación, 21(2), 129139. https://www.viaeducacion.org/downloads/ap/ ehd/aprendizaje_dialogico.pdf

Azorín Abellán, C. (2018). El método de aprendizaje cooperativo y su aplicación en las aulas. Perfiles Educativos, XL(161), 181-194. http://www.scielo.org.mx/pdf/peredu/v40n161/0185-2698-peredu-40-161-181.pdf

Bañales Faz, G., Vega López, N. A., Araujo Alvineda, N., Reyna Valladares, A. y Rodríguez Zamarripa, B. S. (2015). La enseñanza de la argumentación escrita en la universidad. Revista Mexicana de Investigación Educativa, 20(66), 879-910. https://bit.ly/2LLGzeu

Baquero, J. y Pardo, J. F. (1997). La argumentación en el texto jurídico: un instrumento para su comprensión. Ministerio de Justicia y del Derecho.

Barberá, E. (2006). Fundamentos teóricos de la tutoría presencial y en línea: una perspectiva socioconstructivista [En línea]. Educación en Red y Tutoría En Línea, 161-180. https://nanopdf.com/download/ los-fundamentos-teoricos-de-la-tutoria-presencial-y-en-linea_pdf

Brown, K. (2008). Breaking into the tutor's toolbox: an investigation into strategies used in writing center tutorials. University of Louisville. https://bit.ly/2M6CtwR

Calle Álvarez, G. (2016). Perspectiva de los centros de escritura en Colombia. Hallazgos, (28), 145-172. http://www.scielo.org.co/pdf/hall/v14n28/17943841-hall-14-28-00145.pdf

Camps, A. (1995). Aprender a escribir textos argumentativos: características dialógicas de la argumentación escrita. Comunicación, Lenguaje y Educación, (25), 51-63.

https://doi.org/10.1174/021470395321340439

Camps, A. (2004). Objeto, modalidades y ámbitos de la investigación en didáctica de la lengua. Revista Lenguaje, (32), 7-27. https://bit.ly/3277LJe

Cardozo-Ortiz, C. E. (2011). Tutoría entre pares como una estrategia pedagógica universitaria. Educación y Educadores, 14(2), 1-10. http://www.scielo.org. $\mathrm{co} / \mathrm{pdf} / \mathrm{eded} / \mathrm{v} 14 \mathrm{n} 2 / \mathrm{v} 14 \mathrm{n} 2 \mathrm{a} 05 . \mathrm{pdf}$

Carlino, P. (2002). Enseñar a escribir en todas las materias: cómo hacerlo en la universidad. Ponencia en el Simposio Internacional Lectura y Escritura: Nuevos Desafíos. Universidad Nacional de Cuyo, Mendoza, Argentina. https://www.aacademica.org/ paula.carlino/146

Carlino, P. (2006). Representaciones sobre la escritura y formas de enseñarla en universidades de América del Norte. Signos Universitarios, 23(41), 157-186. https://www.aacademica.org/paula.carlino/193

Carlino, P. (2013). Escribir, leer y aprender en la universidad: una introducción a la alfabetización académica. (7. ${ }^{a}$ ed.). Fondo de Cultura Académica. https://www.aacademica.org/paula.carlino/3

Cassany, D. y Castellà, J. (2010). Aproximación a la literacidad crítica. Perspectiva, 28(2), 353-374. https:// doi.org/10.5007/2175-795X.2010v28n2p353

Castelló, M., Bañales Faz, G. y Vega López, N. A. (2010). Enfoques en la investigación de la regulación de escritura académica: estado de la cuestión. Electronic Journal of Research in Educational Psychology, 8(3), 1253-1282. https://doi.org/10.25115/ejrep. v8i22.1424

Castillo, M. (2019). Informe de pasantía: los Cursos Nivelatorios de Lectoescritura. Inédito. Universidad Nacional de Colombia.

Chois-Lenis, P. y Guerrero, H. (2015). Los aportes de un tutor par de escritura académica. En V. Molina (ed.), Panorama de los centros y programas de escritura en Latinoamérica (pp. 53-163). Pontificia Universidad Javeriana. https://www.researchgate. net/publication/308948779_Los_aportes_de_un_ tutor_par_de_escritura_academica 
Chois-Lenis, P., Casas Bustillo, A., López Higuera, A., Prado Mosquera, D. M. y Cajas Paz, E. Y. (2017). Percepciones sobre la tutoría entre pares en escritura académica. Magis, 9(19), 165-184. https://doi.org/10.11144/javeriana.m9-19.ptpe

Díaz, A. (2002). La argumentación escrita. (2. ${ }^{a}$ ed.). Editorial Universidad de Antioquia.

Durán, D. y Flores, M. (2014). Prácticas de tutoría entre iguales en universidades del Estado español y de Iberoamérica. Revista Iberoamericana sobre Calidad, Eficacia y Cambio en Educación, 13(1), 5-17. https://revistas.uam.es/index.php/reice/article/ view/2796/3011

Duran, D., Flores, M., Mosca, A. y Santiviago, C. (2014). Tutoría entre iguales, del concepto a la práctica en las diferentes etapas educativas. InterCambios, 2(1), 31-39. https://grupsderecerca.uab.cat/grai/ sites/grupsderecerca.uab.cat.grai/files/art3_duran. pdf

Freeman, D. (1998). Doing teacher research: From inquiry to understanding. Heinle \& Heinle.

Freire, P. (2002). Pedagogía del oprimido. (3. ${ }^{a}$ ed.). Siglo XXI Editores.

Galeano, M. E. (2004). Estrategias de investigación social cualitativa: el giro en la mirada. La Carreta.

Gallego, Ó. M. y Sánchez, D. L. (2016). Las estrategias tutoriales en los programas de acompañamiento académico en la educación superior: consideraciones generales. En E. Aguirre (ed.), Diálogos 5: discusiones en la psicología contemporánea (pp. 70-90). Universidad Nacional de Colombia. https://www. humanas.unal.edu.co/2017/extension/application/ files/9115/3607/8269/dialogos2017.pdf

Harris, M. (1995). Talking in the middle: Why writers need writing tutors. College English, 57(1), 27-42. https://doi.org/10.2307/378348

Mackiewicz, J. y Thompson, I. (2015). Talk about writing: The tutoring strategies of experienced writing center tutors. Routledge.

Mendieta Izquierdo, G. (2015). Informantes y muestreo en investigación cualitativa. Investigaciones Andina, 17(30), 1148-1150. https://www.redalyc.org/articulo.oa?id=239035878001
Molina, M. E. y Carlino, P. (2013). Escribir y argumentar para aprender: las potencialidades epistémicas de las prácticas de argumentación escrita. Texturas, 13(1-13), 16-32. https://www.aacademica.org/paula.carlino/62.pdf

Molina, V. (2017). Resolución de conflictos en las tutorías de escritura según la teoría pragmadialéctica. Folios, (45), 17-28.

https://doi.org/10.17227/01234870.45folios17.28

Monereo, C. (2008). La enseñanza estratégica: enseñar para la autonomía. En C. Monereo (coord.), Ser estratégico y autónomo aprendiendo. Unidades didácticas de enseñanza estratégica para la ESO (pp. 11-27). Graó. https://www.researchgate.net/ publication/314136312

Núñez Cortés, J. (2013). Una aproximación a los centros de escritura en Iberoamérica. Legenda, 17(17), 64-102. http://erevistas.saber.ula.ve/index.php/ legenda/article/view/4642/4411

Núñez Cortés, J. (2020). Las tutorías de escritura académica: estrategias didácticas de una tutora novel. Enunciación, 25(2), 176-190. https://doi.org/10.14483/22486798.16563

Nussbaum, E. M. (2021). Critical integrative argumentation: Toward complexity in students' thinking. Educational Psychologist, 56(1), 1-17. https://doi.org/10.1080/00461520.2020.1845173

O'Neill, P. (2008). Using peer writing fellows in British universities: Complexities and possibilities. Across the Disciplines, (5), 1-14. doi: https://doi.org/10.37514/atd-j.2008.5.2.06

Pardo, J. F. y Baquero, J. (2001). La estructura argumentativa: base para la comprensión y producción de textos científicos y argumentativos. Forma y Función, (14), 98-118. https://bit.ly/2AYzmk1

Quintana, H. (2004). Manual de tutores. Universidad Interamericana de Puerto Rico.

https://bit.ly/2M1 KbrW

Rapanta, C. (2019). Argumentation strategies in the classroom. Vernon Press.

Red de Lectura y Escritura en Educación Superior (Redlees). (2015). Reflexiones y propuestas para pensar una política de desarrollo de la lectura y la escritura en 
educación superior. En: B. González, A. Salazar-Sierra y L. Peña (comp.), Formación inicial en lectura y escritura en la universidad: de la educación media al desempeño académico en la educación superior (pp. 307-396). Pontificia Universidad Javeriana.

Restrepo, M. (2018). Informe de pasantía como estudiante auxiliar del Programa LEA en la UN. Universidad Nacional de Colombia.

Roldán Morales, C. A. y Arenas Hernández, K. A. (2016). Características de las tutorías del Centro de Lectura y Escritura de la Universidad Autónoma de Occidente: ¿Qué muestran los registros de atención? Grafía, 13(1), 100-114.

https://doi.org/10.26564/16926250.658

Rosales, P. y Vázquez, A. (2011). Leer para escribir y escribir para aprender en la educación superior. Contextos de Educación, 11(11), 1-13. https://www. hum.unrc.edu.ar/publicaciones/contextos/articulos/2011/pdfs/03-rosales-vazquez.pdf

Sala-Bubaré, A. y Castelló, M. (2018). Writing regulation processes in higher education: a review of two decades of empirical research. Reading and Writing, 31(4), 757-777. https://doi.org/10.1007/s11145-017-9808-3

Sampson, V., Grooms, J. y Walker, J. P. (2011). Argument-driven inquiry as a way to help students learn how to participate in scientific argumentation and craft written arguments: An exploratory study. Science Education, 95(2), 217-257. https://doi.org/10.1002/sce.20421

Sanders, J., Wiseman, R. y Gass, R. (2009). Does teaching argumentation facilitate critical thinking? Communication Reports, 7, 27-35.

https://doi.org/10.1080/08934219409367580

Schwarz, B. (2009). Argumentation and learning. En N. Muller Mirza y A. N. Perret-Clermont (eds.), Argumentation and education: Theoretical foundations and practices (pp. 91-126). Springer Science y Business Media B.V.

Serrano de Moreno, S. (2008). Composición de textos argumentativos. Una aproximación didáctica.
Revista de Ciencias Sociales, 14(1), 149-161. https://bit.ly/2M1 subz

Strauss, A. y Corbin, J. (2002). Bases de la investigación cualitativa. Técnicas y procedimientos para desarrollar la teoría fundamentada. Editorial Universidad de Antioquia. https://bit.ly/3277LJe

Tebar, L. (2003). El papel del profesor mediador. Santillana.

Topping, K. J. (1996). The effectiveness of peer tutoring in further and higher education: A typology and review of the literature. Higher Education, 32(3), 321-345.

https://doi.org/10.1007/bf00138870

Toulmin, S., Rieke, R. y Janik, A. (1984). An introduction to reasoning. McMillan Publishing Company.

Vacca, R. y Linek, W. (1992). Escribir para aprender. En J. Irwin y M. A. Doyle (comp.), Conexiones entre lectura y escritura. Aprendiendo de la investigación (pp. 195-214). Aique.

Van Eemeren, F. H. y Grootendorst, R. (2004). A systematic theory of argumentation: The pragma-dialectical approach. Cambridge University Press.

https://doi.org/10.1017/CBO9780511616389

Villagra-Bravo, C. y Valdebenito-Zambrano, V. (2019). Tutoría entre iguales como estrategia para la formación del profesorado. Magis. Revista Internacional de Investigación en Educación, 12(24), 161-176. https://doi.org/10.11144/Javeriana.m12-24.tief

Vygotski, L. (2009). El desarrollo de los procesos psicológicos superiores. (3. a ed.). Editorial Crítica. https://saberespsi.files.wordpress.com/2016/09/vygostki-el-desarrollo-de-los-procesos-psicolc3b3gicos-superiores.pdf

Weston, A. (2006). Las claves de la argumentación. (10. ${ }^{a}$ ed.). Ariel. https:/filosevilla2012.files.wordpress. com/2012/09/lasclavesdeargumentaciona-weston. pdf

Wood, D. J., Bruner, J. S. y Ross, G. (1976). The role of tutoring in problem solving. Journal of Child Psychiatry and Psychology, 17(2), 89-100.

https://doi.org/10.1111/j.1469-7610.1976. tb00381.x

\section{(C) $(1) \Theta(9)$}

